\title{
Effect of self-guided training for the HandbikeBattle on body composition in people with spinal cord injury
}

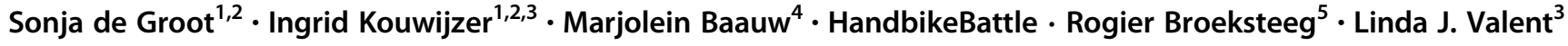

Received: 1 May 2018 / Revised: 18 June 2018 / Accepted: 19 June 2018

(C) International Spinal Cord Society 2018

\begin{abstract}
Study design Single-group pre-test post-test study.

Objectives Examine the change in (1) fitness and body composition due to self-guided training in preparation for the HandbikeBattle in people with spinal cord injury (SCI); and (2) whether these possible body composition changes are associated with changes in fitness.

Setting Handcycling teams of Dutch rehabilitation centers training for the HandbikeBattle, a handcycling race on an Austrian mountain.

Methods Fitness (peak power output (POpeak) determined during a graded handcycling exercise test) and body composition (body mass, body mass index (BMI), waist circumference (WC), \%fat determined with skinfolds and fat and fat free mass (\% and $\mathrm{kg}$ ) assessed by bio-impedance analysis (BIA)) of 18 persons with SCI were evaluated before and after 4 months of training for the HandbikeBattle. The effects of training were evaluated with repeated measures ANOVA. Associations among changes in POpeak and changes in body composition were calculated with Pearson correlation coefficients.

Results Training for the HandbikeBattle led to significant improvements in POpeak, fat mass measured by BIA (19.6 \pm $9.6 \mathrm{~kg}$ to $18.5 \pm 8.3 \mathrm{~kg}, p=0.02)$, \% fat measured by skinfolds $(28.4 \pm 7.8 \%$ to $27.2 \pm 7.2 \%, p=0.02)$, while body mass and WC approached significance $(p=0.06)$. Association among changes in body composition and changes in POpeak ranged from trivial (e.g., for \%fat BIA $r=-0.023$ ) to moderate (e.g., for WC, $r=-0.32$ ).

Conclusions Self-guided training for the HandbikeBattle led to a large increase in fitness but had a small effect on body composition, e.g., nutrition may play a more important role than exercise.
\end{abstract}

The members of HandbikeBattle group are listed below Acknowledgements.

Sonja de Groot

s.d.groot@reade.nl

1 Amsterdam Rehabilitation Research Center I Reade, Amsterdam, The Netherlands

2 University of Groningen, University Medical Center Groningen, Center for Human Movement Sciences, Groningen, The Netherlands

3 Research \& Development, Rehabilitation Center Heliomare, Wijk aan Zee, The Netherlands

4 Nutrition and Dietetics, The Hague University of Applied Sciences, The Hague, The Netherlands

5 Rehabilitation Center Rijndam, Rotterdam, The Netherlands

\section{Introduction}

Being overweight or obese is a significant problem in people with a spinal cord injury (SCI) [1] and is often due to an imbalance between energy expenditure and energy intake. The energy expenditure is often lower in people with $\mathrm{SCI}$, due to a decrease in muscle mass associated with paralysis, an inactive lifestyle and the dependence on arm exercise only [2]. When the daily energy expenditure is lower and there is no change in food intake after injury, this energy imbalance easily leads to becoming overweight or obese in people with SCI.

Besides lowering the energy intake through a proper diet, exercise might help to improve the body composition of (wheelchair-dependent) people with a SCI. To promote regular exercise among people with SCI and other disabilities the HandbikeBattle was conceived, a handcycling race organized yearly on an Austrian mountain for persons previously admitted to Dutch rehabilitation centers [3]. The 
benefits of preparation for such a challenging event was shown in a recent study by the substantial improvements in fitness and health outcome measures over the four months training before the events in 2013 and 2014 [4]. Significant improvements in peak power output (POpeak) $(+17 \%)$ and body composition, i.e., the body mass index (BMI) decreased by $2.1 \%$ and the waist circumference (WC) by $4.1 \%$, were found in this group of 59 relatively inexperienced, recreational handcyclists with self-guided training for four months [4]. However, it was not investigated whether these improvements in fitness and body composition were related. It might be hypothesized that people who train more, improve more in fitness and, parallel to that, improve their body composition.

Although the BMI and WC are cheap and easy to use measures, these measures might not be the best indicators of (changes in) body composition. For example, due to training the fat mass can decrease while the muscle mass increases, and although this is a favorable result, this will not be shown in a change in BMI. WC only focuses on central obesity/abdominal fat and does not take other body areas into account. Body composition measurements like bio-impedance analysis (BIA) and skinfolds give more detail into the body composition and are still feasible to use in the clinic or in large research groups.

Therefore, the present study will be a follow-up of our previous study on the effects of training for the HandbikeBattle on health and fitness including more detailed measures regarding body composition and focusing on people with SCI only [4]. The aims of this study were to investigate in a new cohort: (1) the change in fitness and body composition measured by BIA and skinfolds due to self-guided training in preparation for a handcycling mountain time trial in people with SCI; and (2) whether these possible body composition changes are associated with changes in fitness. The hypothesis is that training for the HandbikeBattle leads to positive changes in body composition and that those who improve more in fitness, improve more in their body composition.

\section{Methods}

The STROBE guidelines were used to ensure the reporting of this prospective cohort study [5].

\section{Participants}

Participants were persons previously admitted to ten Dutch rehabilitation centers that participated in the HandbikeBattle 2016. The participants were recruited by the clinicians of their rehabilitation center, enrolled voluntarily, and were relatively inexperienced handcyclists. A physician medically screened all participants prior to the start of the test and training period. Exclusion criteria were contraindications for exercise according to American College of Sports Medicine guidelines [6] or other medical conditions that did not allow performance of exercise. All included participants with SCI for the HandbikeBattle were asked to participate in the body composition measurements. Because of the BIA measurement, extra exclusion criteria for the present study were having a pacemaker or being pregnant.

\section{Design}

To evaluate the effect of training on the body composition measures, testing occurred before the start of the training period in February (T1) and 4 months later in June, just before the HandbikeBattle event (T2). At T1, personal data (age and sex) and SCI (i.e., lesion level and completeness and time since injury) data were collected. On both test occasions, body composition and fitness were measured with the same methods by the same testers. The body composition measures were tested by one tester, who travelled to the different rehabilitation centers. The exercise test was performed by a tester of the center.

\section{Body composition measurements}

\section{Body mass index}

The participants were asked to report their body height. The body mass was measured on a weighing scale in the rehabilitation center. The BMI was calculated by dividing the body mass $(\mathrm{kg})$ by the squared height $(\mathrm{m})$.

\section{Waist and hip circumference}

The WC was measured twice at the level of the umbilicus with the participants in supine position. The hip circumference was measured twice at the widest point of the hips. The average value of the two measurements was used for analysis. The waist-to-hip ratio was calculated as the ratio of the circumference of the waist to that of the hips.

\section{Skinfolds}

The skinfold measurements were performed with a skinfold caliper (Huidplooimeter Pro, the Netherlands) on the right side of the body. Four skinfolds were measured (biceps, triceps, subscapularis, and supra-iliac) three times according to the ISAK protocol [7] and the average value was used for further analysis for a more reliable outcome [8]. Body density was calculated using the equations from the skinfold equations of Durnin and Womersley [9]. Body fat percentage (\%fat) was then estimated using the Siri equation [10]: 
$\%$ fat $=(4.95 /$ density -4.50$) \times 100$. Although the skinfolds and BIA measurement have the same outcome measure $(\%$ fat), it is much cheaper to perform the skinfolds measurement. However, in contrast to BIA, it is technically a more difficult measure to perform and, therefore, could lead to lower correlations with the change in POpeak.

\section{Bio-impedance analysis}

The BIA was executed with the Bodystat 1500MDD (Bodystat Inc, Douglas, United Kingdom) according to the protocol of Zweers et al. [11], whereby two electrodes were attached to the right foot, at the end of the second metatarsal and between the medial and lateral malleoli of the ankle, and two electrodes were attached to the right hand, at the distal end of the third metacarpal and between the styloid processes of the radius and ulna. The participants were asked not to participate in heavy exercise and to empty the bladder before the measurement. Furthermore, the participant was asked to lie down quietly for a few minutes before the measurement started so that fluid could be evenly distributed over the body. Outcome measures of the BIA were percentage and mass $(\mathrm{kg})$ body fat, percentage and mass $(\mathrm{kg})$ fat free mass, fat mass index $\left(\mathrm{FMI}, \mathrm{kg} / \mathrm{m}^{2}\right)$ and fat free mass index $\left(\mathrm{FFMI}, \mathrm{kg} / \mathrm{m}^{2}\right.$ ). The FFMI and FMI are equivalent concepts to the BMI, i.e., it is the fat free mass or fat mass divided by the squared height, and BMI is the sum of FFMI and FMI. The advantage of the combined use of these indices is that one can judge whether the deficit or excess of body mass is selectively due to a change in FFM vs FM or both combined [12].

\section{Physical capacity}

The physical capacity was determined in the rehabilitation centers on an arm crank ergometer (Lode Angio, Groningen, the Netherlands), or with the personal handcycle on a roller system (Tacx, Terneuzen, the Netherlands) or on the Cyclus2 ergometer (RBM elektronik-automation $\mathrm{GmbH}$, Leipzig, Germany). Participants were asked to empty their bladder before testing. The protocol used was similar between centers [3]. The exercise test was preceded by a 5 min resting period. After a warm-up, the graded exercise test was performed. The starting workload of the graded exercise test was dependent on the handcycling classification and (estimated) handcycling level of the participants and was set between $20-30 \mathrm{~W}$ for the lower level handcyclist and between 40-60 W for the higher level handcyclists. The workload was increased every minute by $8-10$ Watt (lower level) and 15-20 Watt (higher level). POpeak was the highest power output that was maintained for at least $30 \mathrm{~s}$.

\section{Training}

At T2, participants reported their performed training over the last four months (i.e., between $\mathrm{T} 1$ and $\mathrm{T} 2$ ) by filling-out a representative training week with the type of training and duration for every session. Furthermore, they were asked whether they had changed their lifestyle between $\mathrm{T} 1$ and $\mathrm{T} 2$. Their potential training time was calculated as the number of days between the tests at $\mathrm{T} 1$ and $\mathrm{T} 2$.

\section{Statistics}

Descriptives were calculated for all characteristics and outcome measures. The assumption of normality was checked for the outcome measures with a Shapiro-Wilk test and by visual inspection of the q-q plot.

To test for baseline (T1) differences between the group that was tested twice (at $\mathrm{T} 1$ and $\mathrm{T} 2$ ) and the group that did not participate at T2 an independent sample $t$-test (for age and body composition measures) and a Chi-squared test (for sex) were used.

To assess the effect of handcycling training on POpeak and body composition outcomes repeated measurements ANOVA was performed with test occasion (T1 and T2) as within factor.

Associations among changes in POpeak or absolute weekly training hours or potential training time between test occasions and changes in body composition measures were calculated with Pearson correlation coefficients. Correlation coefficients were considered trivial when between $0.0-0.1$, small between $0.1-0.3$, moderate between $0.3-0.5$, large between $0.5-0.7$, very large between $0.7-0.9$, and nearly perfect between 0.9-1.0 [13]. To be able to correct for the fitness level at $\mathrm{T} 1$, a linear regression analysis was also performed with the change in body composition outcome as dependent variable, the change in POpeak as independent variable, and the absolute POpeak at T1 was added to correct for the basic fitness level. Significance was set at $p<$ 0.05 for all statistical analyses. IBM SPSS statistics 20 (IBM Corporation, New York, USA) was used for all analyses.

\section{Results}

\section{Descriptives}

Thirty handcyclists with SCI were included and tested at T1. However, 18 of them also participated at the second test occasion. Reasons for drop-out were: not participating in the HandbikeBattle anymore due to e.g., illness or not enough time to train, not able to plan a second time for the 
Table 1 Descriptives of the body composition measures are shown, together with the effect of training on the body composition ( $p$ and effect size), the responsiveness (standardized response mean (SRM)), and the correlation coefficient between the changes in body composition and the change in peak power output ( $\Delta$ POpeak)

\begin{tabular}{|c|c|c|c|c|c|c|c|}
\hline & $\mathrm{N}$ & $\begin{array}{l}\text { T1 } \\
\text { Mean (SD) }\end{array}$ & $\begin{array}{l}\mathrm{T} 2 \\
\text { Mean (SD) }\end{array}$ & \%change & $p$ & $\begin{array}{l}\text { Effect size } \\
\text { Cohen's d }\end{array}$ & $\begin{array}{l}\text { Correlation with } \triangle \text { POpeak } \\
r\end{array}$ \\
\hline Body mass $(\mathrm{kg})$ & 18 & $77.4(17.3)$ & $76.1(16.5)$ & $-1.7 \%$ & 0.063 & 0.08 & -0.077 \\
\hline Waist circumference $(\mathrm{cm})$ & 18 & $89.8(14.7)$ & $88.5(13.4)$ & $-1.4 \%$ & 0.059 & 0.09 & -0.322 \\
\hline Hip circumference $(\mathrm{cm})$ & 18 & $91.6(11.8)$ & $90.3(11.6)$ & $-1.4 \%$ & 0.038 & 0.11 & -0.164 \\
\hline Waist Hip ratio & 18 & $0.98(0.05)$ & $0.98(0.04)$ & $0 \%$ & 0.845 & 0 & -0.168 \\
\hline Body mass index $\left(\mathrm{kg} / \mathrm{m}^{2}\right)$ & 18 & $24.3(4.9)$ & $24.3(5.0)$ & $0 \%$ & 0.680 & 0 & -0.158 \\
\hline Fat mass index $\left(\mathrm{kg} / \mathrm{m}^{2}\right)$ & 18 & $6.3(3.4)$ & $5.9(3.0)$ & $-6.3 \%$ & 0.028 & 0.12 & -0.006 \\
\hline Fat free mass index $\left(\mathrm{kg} / \mathrm{m}^{2}\right)$ & 18 & $18.1(2.9)$ & $18.0(2.9)$ & $-0.006 \%$ & 0.737 & 0.03 & -0.058 \\
\hline$\%$ Fat BIA $(\%)$ & 18 & $24.7(9.2)$ & $24.0(8.4)$ & $-2.8 \%$ & 0.132 & 0.08 & -0.023 \\
\hline Fat BIA (kg) & 18 & $19.6(9.6)$ & $18.5(8.3)$ & $-5.6 \%$ & 0.020 & 0.11 & -0.041 \\
\hline$\%$ Fat free mass BIA $(\%)$ & 18 & $75.3(9.2)$ & $76.0(8.4)$ & $+0.01 \%$ & 0.132 & 0.08 & 0.023 \\
\hline Fat free mass BIA $(\mathrm{kg})$ & 18 & $57.8(12.4)$ & $57.7(12.4)$ & $-0.001 \%$ & 0.775 & 0.008 & -0.066 \\
\hline$\%$ Fat skinfolds (\%) & 18 & $28.4(7.8)$ & $27.2(7.2)$ & $-4.2 \%$ & 0.019 & 0.15 & -0.116 \\
\hline POpeak (W) & 16 & $110(37)$ & $129(45)$ & $+17 \%$ & $<0.001$ & 0.51 & - \\
\hline
\end{tabular}

tests. The 18 handcyclists did not differ significantly regarding sex $(p=0.67)$, age $(p=0.68)$, and body composition $(p=0.49-0.98)$ at T1 compared to the 12 participants who dropped-out.

Of the 18 handcyclists who completed both test occasions, $72 \%$ were male and their age was on average $39.1 \pm$ 12.2 years, height $1.78 \pm 0.09 \mathrm{~m}$ and median time since injury 3 years (interquartile range (IQR): 1.4-10.0). Of these 18 participants, four had a cervical SCI, ten had a thoracic SCI and four had a SCI at the lumbar level. Nine of 18 participants had a motor complete lesion. The average time between test occasions was $158 \pm 32$ days. Descriptives of body composition outcome measures and POpeak are shown in Table 1. Twelve participants reported a representative training week with a median of three times a week (IQR: 2-3) and a median of $6 \mathrm{~h} /$ week (IQR: 5-7.5). The other six participants did not complete the requested logs. Three of 13 participants reported that they changed their diet, of whom one explicitly said that the goal was to lose weight.

\section{Effect of training on fitness and body composition measures}

The effect of the ANOVA demonstrated a significant decrease over the training period for hip circumference ( $p$ $=0.038)$, \% fat based on the skinfolds $(p=0.019)$, fat mass measured by BIA $(\mathrm{kg})(p=0.020)$, and the FMI $(p=0.028)$, while body mass $(p=0.063)$ and WC approached significance (0.059)(Table 1). POpeak increased significantly by $17 \%$ between $\mathrm{T} 1$ and T2 $(p<0.001$; Table 1$)$. Effect sizes ranged from small to medium. Figure 1 shows the individual results of BMI, WC, \%fat measured by BIA, and $\%$ fat measured by the skinfolds.

Association among changes in body composition and changes in POpeak ranged from trivial (e.g., for FMI $r=$ -0.006 ) to moderate (e.g., for WC, $r=-0.32$ ). All correlations are shown in Table 1. Figure 2 shows the trivial relationship between the change in POpeak and the change in \%fat measured by BIA. The linear regression analyses, with a correction for POpeak at T1, did not show any significant relationship between the change in POpeak and change in body composition measure over the training period. The association among changes in body composition and weekly training hours (in $N=12$ ) was trivial to moderate, varying from $r=0.014$ (WC) to $r=0.385$ (\% fat measured by skinfolds). Similar results were found for the association among changes in body composition and potential training time between test occasions.

\section{Discussion}

In summary, self-guided training for the HandbikeBattle led to significant but small to medium improvements in body composition measures (up to 5.6\%) while the fitness level improved much more (17\%). These small changes in body composition did not seem to relate to changes in fitness level.

The BMI of our group $\left(24.3 \mathrm{~kg} / \mathrm{m}^{2}\right)$ was quite similar to the BMI at T1 of the HandbikeBattle participants of 2013 and $2014\left(24.1 \mathrm{~kg} / \mathrm{m}^{2}\right)$ [4]. However, the participants in the study of Hoekstra et al. [4] significantly decreased their BMI by $0.50 \mathrm{~kg} / \mathrm{m}^{2}$ in contrast to our study. The previous 

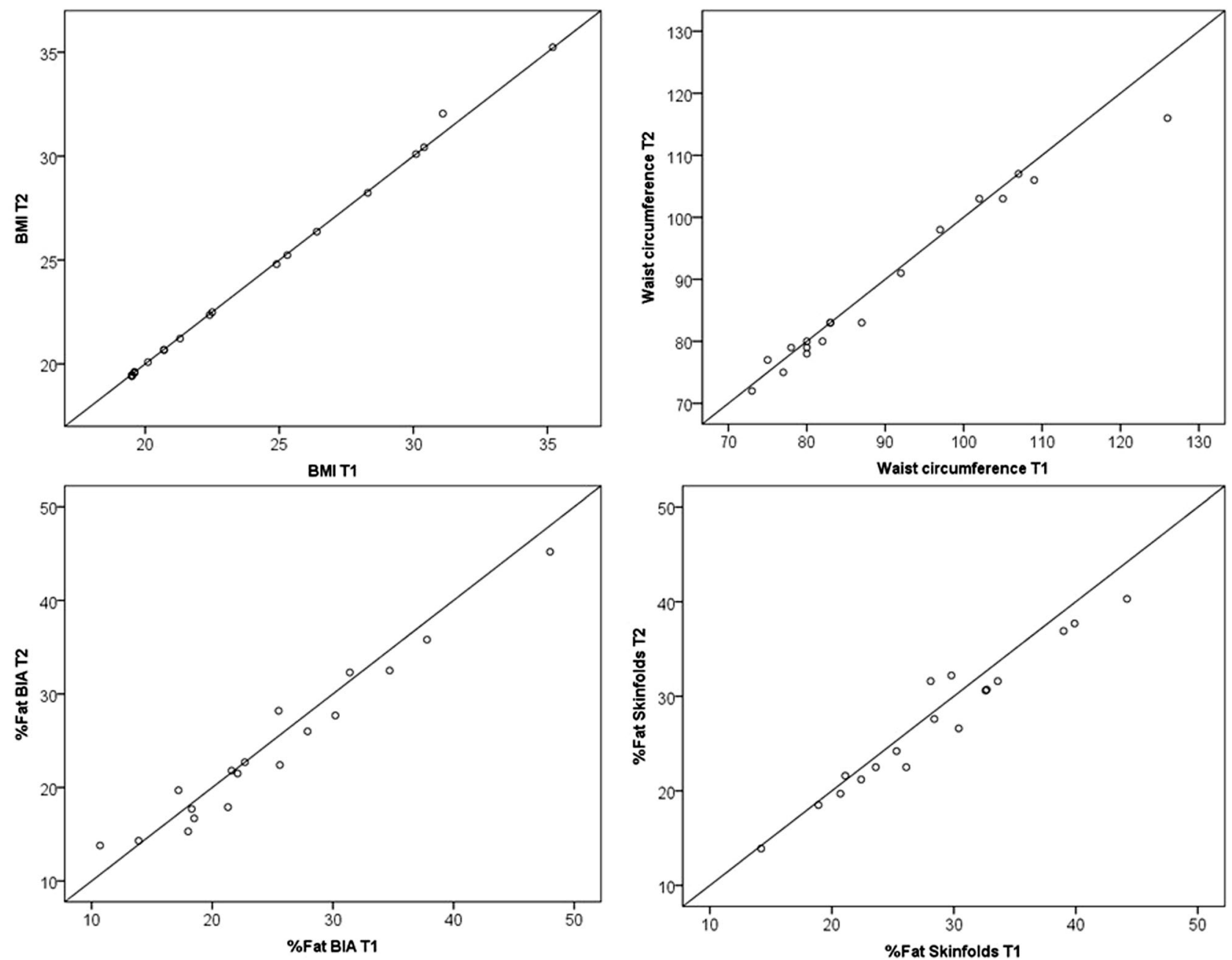

Fig. 1 Individual results of the BMI, waist circumference, \%fat BIA and \%fat at the first (T1) and second (T2) test occasion. Each dot represents the outcome of one participant. The diagonal line is the line

of identity. For example, if a data point lies above the line in the lower left graph, this means that the \%Fat measured by BIA is lower at the second test occasion

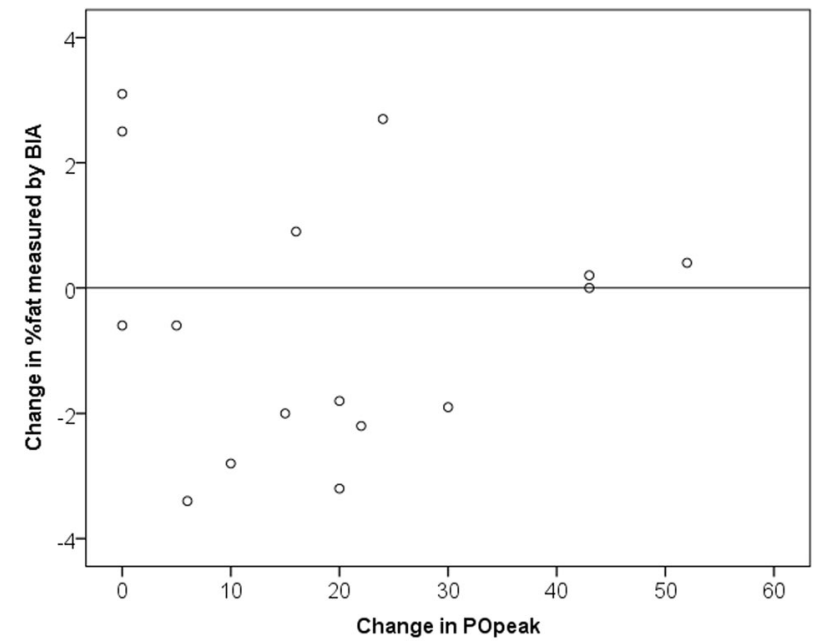

Fig. 2 Relationship between change in peak power output (POpeak) and change in \%fat measured by bio-impedance analysis (BIA) $(r=$ $-0.023)$

HandbikeBattle study [4] showed a significant decrease in WC while the current HandbikeBattle study showed a smaller decrease which approached significance $(-3.7 \mathrm{~cm}$ vs. $-1.3 \mathrm{~cm})$. These differences between the studies do not seem to be explained by the reported training hours per week (past study [4]: $6.7 \pm 3.4 \mathrm{~h} /$ week; present study: $6.2 \pm$ $2.1 \mathrm{~h} /$ week). Differences in the change in BMI and WC over time between the two studies might be explained by the smaller sample size and the focus on people with a SCI only in the present study.

When compared to a cross-sectional study among 223 Dutch persons with SCI (TSI $>10$ years), the BMI of our group was slightly higher than the group without metabolic syndrome $\left(23.5 \mathrm{~kg} / \mathrm{m}^{2}\right)$ [14]. The body composition of our group of handcyclists showed on average unfavorable values compared to wheelchair athletes in the literature. The BMI $\left(20.8-22.5 \mathrm{~kg} / \mathrm{m}^{2}\right)$, WC $(67.8-75.4 \mathrm{~cm})$ and waist/hip ratio (0.87-0.95) in wheelchair athletes with SCI [15] were 
much lower than in our group. Goosey-Tolfrey et al. [16] measured the percentage fat mass by, among others, skinfolds (19.1\% fat mass) and BIA ( $18.4 \%$ fat mass) in 30 wheelchair games players and found $6-10 \%$ lower values. These results indicated that the body composition of the participants of the HandbikeBattle is mostly comparable to the body composition of general wheelchair users with SCI.

As far as known, the FMI and FFMI have not been reported in people with SCI in previous literature but might be interesting due to the changes in body composition as a consequence of paralysis. During the self-guided training period the FMI decreased significantly while the FFMI did not change. However, when the FFMI and FMI of our handcyclists are compared to reference values of ablebodied persons [12], our values seem to fall within the normal ranges.

The training period for the HandbikeBattle induced also significant but only small changes in hip circumference, fat mass measured by BIA and \%fat mass measured by skinfolds. In contrast, no changes were found in BMI, waist/hip ratio, and fat free mass. Previous studies, under standardized (lab-based) handcycling or arm crank training conditions, showed contradictory results. Some studies found improvements in some of the body composition measures, like BMI [4, 17], WC [4, 17-19], trunk, and android fat percentage [18]. However, other studies did not find changes in \%fat measured by skinfolds [20] or BIA [17] after an eight $(5 \times / \mathrm{wk}, 30 \mathrm{~min})$ or six weeks $(3 \times / \mathrm{wk}, 60 \mathrm{~min})$ arm ergometer or handcycling training program, respectively. Furthermore, results of a review by Fisher et al. [21] also showed that there is lack of evidence supporting the benefits of upper-extremity resistance circuit training (combination of weight training and aerobic training) on body composition in people with SCI.

While the fitness level increased with $17 \%$ during the training period for the HandbikeBattle, the \%fat, for example, decreased with a much lower percentage of $2.8-4.2 \%$. Only weak to moderate relationships between changes in fitness level and changes in body composition measures were found in our recreational handcyclists with a SCI, indicating that the effect of training on fitness level was different than the effect on body composition. So, although significant changes have been found in body composition in the present study and in previous studies, exercise does not seem to have an unambiguous effect on body composition. Besides, we cannot conclude from most of these studies that these possible favorable changes in body composition are only due to the exercise program since other lifestyle change (i.e., dietary changes) could have played a role as well as also indicated by three of our participants.

Another limitation of the present study then previously mentioned, is the lack of a control group. For studying the associations between changes in body composition and change in fitness level over time, no control group is necessary. The changes in body composition that were found over time were just small and, therefore, not of great clinical relevance. Furthermore, due to the specific inclusion criteria and the motivation to participate in a challenging handcycling event as the HandbikeBattle, we probably cannot generalize the results to all people with a SCI. The limited information on the self-guided training of the participants makes it difficult to draw strong conclusions about the association between training doses and improvements in fitness and body composition. However, the aim of this study was to explore whether self-guided training for the HandbikeBattle had an effect on fitness and body composition.

In conclusion, training for the HandbikeBattle led to significant but only small to medium improvements in body composition. Furthermore, only weak to moderate relationships between changes in fitness level and changes in body composition measures were found. This might implicate that a self-guided handcycling training program does not have a large effect on the body composition and that other factors, i.e., nutrition or training with a higher volume may play a more important role in changing the body composition. Future research could focus on the effect of nutrition, possibly combined with different training protocols, on body composition.

Acknowledgements The assistance of Suzanne de Groot during part of the data collection is greatly appreciated.

\section{Compliance with ethical standards}

Conflict of interest The authors declare that they have no conflict of interest.

Statement of ethics The study was approved by the Local Ethical Committee of the Center for Human Movement Sciences, University Medical Center Groningen, the Netherlands. All participants voluntary signed an informed consent form after they were given information about the testing procedures. The study was registered in the Dutch Trial Register (www.trialregister.nl, NTR6586).

\section{HandbikeBattle group name}

Bram van Gemeren, Rehabilitation center Het Roessingh, Enschede, The Netherlands

Mark van de Mijll Dekker, Rehabilitation center Heliomare, Wijk aan Zee, The Netherlands

Karin Postma, Rehabilitation Center Rijndam, Rotterdam, The Netherlands

Linda van Vliet, Reade center for rehabilitation \& rheumatology, Amsterdam, The Netherlands

Misha Metsaars, Libra Rehabilitation \& Audiology, location Blixembosch, Eindhoven, The Netherlands 
Eric Helmantel, University Medical Center Groningen, Location Beatrixoord, Haren, The Netherlands

Lise Wilders, Sint Maartenskliniek, Nijmegen, The Netherlands

Maremka Zwinkels, Rehabilitation center De Hoogstraat, Utrecht, The Netherlands

Paul Grandjean, Rehabilitation center Adelante, Hoensbroek, The Netherlands

Selma Overbeek, Rehabilitation center Tolbrug, Den Bosch, The Netherlands

\section{References}

1. de Groot S, Post M, Postma K, Sluis T, van der Woude L. Prospective analysis of body mass index during and up to 5 years after discharge from inpatient spinal cord injury rehabilitation. $\mathbf{J}$ Rehabil Med. 2010;42:922-8.

2. Sedlock DA, Laventure SJ. Body composition and resting energy expenditure in long term spinal cord injury. Spinal Cord. 1990;28:448-54.

3. de Groot S, Postma K, van Vliet L, Timmermans R, Valent LJM. Mountain time trial in handcycling: exercise intensity and predictors of race time in people with spinal cord injury. Spinal Cord. 2014;52:455-61.

4. Hoekstra S, Valent L, Gobets D, van der Woude L, de Groot S. Effects of four-month handbike training under free-living conditions on physical fitness and health in wheelchair users. Disabil Rehabil. 2017;39:1581-8.

5. von Elm E, Altman DG, Egger M, Pocock SJ, Gøtzsche PC, Vandenbroucke JP, et al. The Strengthening the Reporting of Observational Studies in Epidemiology (STROBE) statement: guidelines for reporting observational studies. J Clin Epidemiol. 2008;61:344-9.

6. American College of Sports Medicine. Guidelines for exercise testing and prescription. (Wolters Kluwer/Lippincott Williams \& Wilkins Health, 1991).

7. Stewart AA, Marfell-Jones M, Olds T, Al E. International standards for anthropometric assessment. Low Hutt, New Zeal Int Soc Adv Kinanthropometry. 2011; 125f. http://www.ceap.br/material/ MAT17032011184632.pdf. Accessed 25 Apr 2018.

8. de Vet HC, Terwee CB, Mokkink LB, Knol DL. Measurment in medicine. A practical guide. Cambridge: Cambridge University Press; 2011.
9. Durnin JV, Womersley J. Body fat assessed from total body density and its estimation from skinfold thickness: measurements on 481 men and women aged from 16 to 72 years. Br J Nutr. 1974;32:77-97.

10. SIRI WE. The gross composition of the body. Adv Biol Med Phys. 1956;4:239-80.

11. Zweers H, Kruizenga H, Berg A van den, Reijven N, Hulshof P. Single Frequency Bio-Impedantie Analyse, Standard Operating Procedures. Nutritional Assessment Platform. 2016, http://za kboekdietetiek.nl/wp-content/uploads/2016/03/NAP-BIA-SOP. pdf. Accessed 25 Apr 2018.

12. Schutz Y, Kyle U, Pichard C. Fat-free mass index and fat mass index percentiles in Caucasians aged 18-98 y. Int $\mathrm{J}$ Obes. 2002;26:953-60.

13. Hopkins WG. A scale of magnitudes for effect statistics. Sport Sci. 2002;5:1-7.

14. de Groot S, Adriaansen JJ, Tepper M, Snoek GJ, van der Woude LHV, Post MWM. Metabolic syndrome in people with a longstanding spinal cord injury: associations with physical activity and capacity. Appl Physiol Nutr Metab. 2016;41:1190-6.

15. Mojtahedi MC, Valentine RJ, Evans EM. Body composition assessment in athletes with spinal cord injury: comparison of field methods with dual-energy X-ray absorptiometry. Spinal Cord. 2009;47:698-704.

16. Goosey-Tolfrey V, Keil M, Brooke-Wavell K, de Groot S. A comparison of methods for the estimation of body composition in highly trained wheelchair games players. Int J Sports Med. 2016;37:799-806.

17. Kim D-I, Lee H, Lee B-S, Kim J, Jeon JY. Effects of a 6-week indoor hand-bike exercise program on health and fitness levels in people with spinal cord injury: a randomized controlled trial study. Arch Phys Med Rehabil. 2015;96:2033-40.e1.

18. Bakkum A, Paulson T, Bishop N, Goosey-Tolfrey V, StolwijkSwüste J, Kuppevelt D, et al. Effects of hybrid cycle and handcycle exercise on cardiovascular disease risk factors in people with spinal cord injury: a randomized controlled trial. J Rehabil Med. 2015;47:523-30.

19. Rosety-Rodriguez M, Camacho A, Rosety I, Fornieles G, Rosety MA, Diaz AJ, et al. Low-grade systemic inflammation and leptin levels were improved by arm cranking exercise in adults with chronic spinal cord injury. Arch Phys Med Rehabil. 2014;95:297-302.

20. Taylor AW, McDonell E, Brassard L. The effects of an arm ergometer training programme on wheelchair subjects. Spinal Cord. 1986;24:105-14.

21. Fisher JA, McNelis MA, Gorgey AS, Dolbow DR, Goetz LL. Does upper extremity training influence body composition after spinal cord injury? Aging Dis. 2015;6:271-81. 